\title{
NONAMENABILITY AND BOREL PARADOXICAL DECOMPOSITIONS FOR LOCALLY COMPACT GROUPS
}

\author{
ALAN L. T. PATERSON
}

\begin{abstract}
We show that a locally compact group $G$ is not amenable if and only if it
\end{abstract} admits a Borel paradoxical decomposition.

In 1938 A. Tarski [7] proved the following remarkable theorem. Let $G$ be a group acting invertibly on a set $X$ and $A \subset X$. Then there exists a positive, finitely additive, $G$-invariant measure $\mu$ on $X$ with $\mu(A)=1$ if and only if $A$ does not admit $a$ paradoxical decomposition. Here, a subset $B$ of $X$ admits a paradoxical decomposition (p.d.) if there exists a partition $A_{1}, \ldots, A_{m}, B_{1}, \ldots, B_{n}$ of $B$ and elements $x_{1}, \ldots, x_{m}, y_{1}, \ldots, y_{n}$ of $G$ such that both $\left\{x_{i} A_{i}: 1 \leqslant i \leqslant m\right\}$ and $\left\{y_{i} B_{i}: 1 \leqslant i \leqslant n\right\}$ are partitions of $B$. (Thus, by using $G$-translates, we can "pack" two copies of $B$ into itself.) In the above circumstances it is convenient to say that $A_{i}, B_{i}, x_{i}, y_{i}$ is a p.d. (for $B$ with respect to $G$ ). An immediate consequence of Tarski's theorem is that $a$ (discrete) group $G$ is not amenable if and only if $G$ admits a p.d. This beautiful result thus characterizes amenability directly in terms of translates of subsets of $G$ with no mention of invariant means or measures. Tarski's proof uses a deep set-theoretic result of D. König [3]. Is there a simpler proof available?

A natural question, raised by W. R. Emerson, is the topological analogue of the above nonamenability theorem. Let $G$ be a locally compact group. Let us say that $G$ admits a Borel p.d. if there exists a p.d. as above with every $A_{i}, B_{i}$ a $B$ orel subset of $G$. The question then is: Is it true that $G$ is not amenable if and only if $G$ admits $a$ Borel p.d.? The object of this note is to show that the answer to this question is yes.

What about a topological analogue for Tarski's theorem? The reader is referred to [2] for information about amenable locally compact groups.

THEOREM. Let $G$ be a locally compact group. Then $G$ is not amenable if and only if $G$ admits a Borel p.d.

Proof. Trivially, if $G$ admits a Borel p.d., then $G$ is not amenable. Conversely, suppose that $G$ is not amenable. Since $G$ is the (directed) union of its $\sigma$-compact, open subgroups, there exists a $\sigma$-compact, nonamenable, open subgroup $H$ of $G$. Suppose that the result is true for $H$, and let $A_{i}, B_{i}, x_{i}, y_{i}$ be a Borel p.d. for $H$ as above. Let $T$ be a transversal for the right $H$-cosets in $G$. One readily checks that $A_{i} T, B_{i} T, x_{i}, y_{i}$ is a p.d. for $G$. To show that this p.d. is Borel, we need only show

Received by the editors November 28, 1984.

1980 Mathematics Subject Classification. Primary 43A07, 22D05.

(c) 1986 American Mathematical Society $0002-9939 / 86 \$ 1.00+\$ .25$ per page 
that $A T$ is Borel in $G$ if $A$ is Borel in $H$. This is obvious if $A$ is open in $H$, since then $A T$ is open in $G$, and the result for general $A$ follows by using the monotone class lemma. (Note that if $\left\{C_{n}\right\}$ is a decreasing sequence of subsets of $H$, then $\bigcap_{n=1}^{\infty}\left(C_{n} T\right)=\left(\cap_{n=1}^{\infty} C_{n}\right) T$. $)$

Thus $G$ admits a Borel p.d., so we can suppose that $G=H$-i.e., $G$ is $\sigma$-compact.

Since $G$ is $\sigma$-compact, we can find a compact, normal subgroup $K$ of $G$ with $G / K$ separable. Since $K$ is amenable and $G$ is not amenable, we have $G / K$ not amenable. Let $Q: G \rightarrow G / K$ be the quotient map. If there exists a Borel p.d. involving sets $A_{i}$, $B_{i}$, then, by considering $Q^{-1}\left(A_{i}\right), Q^{-1}\left(B_{i}\right)$, we see that $G$ admits a Borel p.d. We can therefore suppose that $G$ is separable.

Let $G_{e}$ be the identity component of $G$. Then $G / G_{e}$ is totally disconnected, and so contains a compact open subgroup $L$. Let $\Phi: G \rightarrow G / G_{e}$ be the quotient map and $H=\Phi^{-1}(L)$. Then $H$ is an almost connected, open and closed subgroup of $G$. There are two cases to be considered.

(i) $H$ is not amenable. A result of Rickert $[5,6]$ shows that there exists a discrete subgroup $F$ of $H$ isomorphic to the free group $F_{2}$ on two generators. In particular, $F$ is closed in $H$. Now $H$ is separable since $G$ is, and a result of [4] yields a Borel cross section $B$ for the right $F$-cosets in $H$. Now $F$ is, of course, not amenable, and so by Tarski's theorem, we can find a p.d. $A_{i}^{\prime}, B_{i}^{\prime}, x_{i}, y_{i}$ for $F$. Then $A_{i}^{\prime} B, B_{i}^{\prime} B, x_{i}, y_{i}$ is a p.d. for $H$, and the p.d. is Borel since each $A_{i}^{\prime}, B_{i}^{\prime}$ is countable and $B$ is Borel. We then produce a Borel p.d. for $G$ as in the second paragraph of the present proof.

(ii) $H$ is amenable. The group $G$ acts on the discrete space $G / H$ in the usual way. We claim that there does not exist a $G$-invariant mean on $\ell_{\infty}(G / H)$. (Indeed, following the usual line of argument in this context, if $m$ were such a mean, and $n$ was a left invariant mean on the space $C(H)$ of bounded, continuous, complexvalued functions on $H$, then the map $\phi \rightarrow m\left(x H \rightarrow n\left(\left.(\phi x)\right|_{H}\right)\right)$, where $\phi x(y)=$ $\phi(x y)(x, y \in G)$, is a left invariant mean on $C(G)$, giving $G$ amenable and, hence, a contradiction.) By Tarski's theorem we can find a p.d. $A_{i}, B_{i}, x_{i}, y_{i}$ for $G / H$ with respect to $G$. Then $A_{i} H, B_{i} H, x_{i}, y_{i}$ is a Borel p.d. for $G$, and we are finished.

\section{REFERENCES}

1. W. R. Emerson, The Hausdorff paradox for general group actions, J. Funct. Anal. 32 (1979), 213-227.

2. F. P. Greenleaf, Invariant means on topological groups, Van Nostrand, New York, 1969.

3. D. König, Sur les correspondences multivoques des ensembles, Fund. Math. 8 (1926), 114-134.

4. G. W. Mackey, Induced representations of locally compact groups. I, Ann. of Math. (2) 55 (1952), $101-139$.

5. N. W. Rickert, Some properties of locally compact groups, J. Austral. Math. Soc. 7 (1967), $43 \hat{j}-454$.

6. Amenable groups and groups with the fixed point property, Trans. Amer. Math. Soc. 127 (1967), 221-232.

7. A. Tarski, Algebraische Fassung des Massproblems, Fund. Math. 31 (1938), 47-66.

Department of Mathematics, University of Western Ontario, London, Canada N6A 5B7

Current address: Department of Mathematics, The Edward Wright Building, Dunbar Street, University of Aberdeen, Aberdeen, Scotland, U.K. 